\title{
Dissecting Aneurysm of the Anterior Temporal Artery -Case Report-
}

\author{
Katsuya UMEOKA, ${ }^{1}$ Kazutaka SHIROKANE, ${ }^{1}$ Takayuki MizUNARI, ${ }^{1}$ \\ Shiro KOBAYASHI, ${ }^{1}$ and Akira TERAMOTO ${ }^{2}$ \\ ${ }^{1}$ Department of Neurosurgery, Nippon Medical School Chiba Hokusoh Hospital, Inzai, Chiba; \\ ${ }^{2}$ Department of Neurosurgery, Nippon Medical School, Tokyo
}

\begin{abstract}
A 65-year-old woman presented a rare dissecting aneurysm of the anterior temporal artery (ATA) manifesting as headache. Computed tomography and magnetic resonance imaging revealed a mixeddensity mass in the horizontal segment of the middle cerebral artery. Emergent angiography demonstrated aneurysmal dilatation and a thrombosed mass in the sylvian fissure. Infectious aneurysm was excluded. She underwent emergent surgery to reduce the risk of repeated infarction and hemorrhage. The distal side of the ATA manifested occlusive changes suggestive of arterial dissection. The proximal side of the ATA was ligated and the lesion was excised. Histological examination confirmed that the aneurysmal dilatation was attributable to arterial dissection due to disruption of the internal elastic lamina. Distal dissecting aneurysms may occur in the absence of infectious disease. We recommend that ruptured distal dissecting aneurysms be treated surgically in the acute stage immediately after detection.
\end{abstract}

Key words: anterior temporal artery, distal middle cerebral artery, dissecting aneurysm, cerebral infarction, proximal ligation

\section{Introduction}

Distal intracerebral aneurysms usually occur as a result of head trauma, vasculitis, bacterial infection, or atherosclerosis. ${ }^{7,10)}$ Arterial dissection was involved in the development of aneurysms unrelated to branching zones, but the specific cause remained unidentified in most patients with intracerebral dissecting aneurysms. ${ }^{12,14)}$ Spontaneous middle cerebral artery (MCA) dissection is relatively rare, and is usually located in the M1 portion. ${ }^{15)}$ True anterior temporal artery (ATA) dissecting aneurysms are extremely rare, with only one reported case. ${ }^{17)}$ We report a rare case of dissecting aneurysm of the ATA manifesting as cerebral infarction.

\section{Case Report}

A 65-year-old woman experienced headache and slight nausea 7 days before admission. She had no clear history of trauma, infection, or systemic angitis; and neither smoked nor drank alcohol. Hematological and laboratory studies revealed no abnormalities. Computed tomography and magnetic resonance imaging performed on admission disclosed subacute infarction in the left ATA territory (Fig. 1). Emergent angiography demonstrated an aneurys- mal dilatation and a thrombosed mass in the horizontal segment of the MCA, but no aneurysm neck was identified (Fig. 2). We planned surgery to reduce the risk of repeated infarction and hemorrhage under a diagnosis of thrombosed aneurysm of the $\mathrm{M}_{1}$ portion of the MCA and infarction of the ATA area due to artery-to-artery embolization from the thrombosed aneurysm.

Left temporal craniotomy was performed. The sylvian fissure was exposed under the operating microscope, and the left internal carotid artery, the $\mathrm{A}_{1}$ portion of the left anterior cerebral artery, the $M_{1}$ portion, and a thrombosed aneurysm were observed (Fig. 3A). The thrombosed aneurysm at first appeared to adhere to and originate at the $M_{1}$, but after sharp dissection, the $M_{1}$ portion was free of aneurysmal dilatation and no findings were indicative of arterial dissection. The ATA was dilated $10 \mathrm{~mm}$ from the origin of the $M_{1}$. The dissection was located in the ATA and flow distal to the lesion was reduced. The proximal portion of the ATA was ligated and the thrombosed mass resected (Fig. 3B). She developed no new neurological deficits or additional ischemic lesions. Histological study revealed dissection of the internal elastic lamina, and elastica-van Gieson staining demonstrated that the ruptured arterial dilatation was located between layers of the internal elastic lamina (Fig. 4). 



Fig. 1 Diffusion-weighted (A), $T_{1}$-weighted (B), $T_{2}$-weighted (C), and fluid-attenuated inversion recovery (D) magnetic resonance images on admission demonstrating subacute infarction in the left anterior temporal artery territory and a thrombosed mass in the left sylvian fissure.


Fig. 2 Anteroposterior (A) and lateral (B) emergent cerebral angiograms and three-dimensional digital subtraction angiogram (C) of the left internal carotid artery demonstrating an aneurysmal dilatation and a thrombosed mass in the horizontal segment of the middle cerebral artery.

\section{Discussion}

Aneurysms located on a distal arterial branch tend to rupture and re-bleed, and the size at the time of rupture may in part be determined by the thickness and diameter of the parent artery. ${ }^{13,16)}$ Since distal branch arteries are thinner with smaller diameter, distal branch aneurysms such as distal MCA aneurysms carry higher risk for rupture.

Thirteen cases of dissecting distal MCA aneurysms have been reported, and 9 cases were admitted with subarachnoid hemorrhage. ${ }^{1-3,5,6,8,9,11,14,17-20)}$ Study of the mechanisms of ruptured/unruptured arterial dissection using immunohistochemical findings of lysyl oxidase (Lox) and matrix metalloproteinases (MMPs) in multiple dissecting aneurysms demonstrated that Lox1, MMP-2, and MMP-9 were remarkably expressed in hypertrophic media outside the intima in ruptured dissecting aneurysms, but were expressed in the intima and inside hypertrophic media in unruptured dissecting aneurysms. ${ }^{17)}$

Our patient was hospitalized with ischemia. We suggest that in arteries that are tethered by a dural attachment just proximal to the dissection site (such as the internal carotid artery or vertebral artery), pulsatile and expansile forces are dissipated by vessel wall failure, resulting in a higher incidence of clinically significant hemorrhage. ${ }^{4)}$ In arteries not tethered in the perilesional area, the pulsatile and expansile forces in a growing dissection may be dissipated by elongation and the development of ectasia, with grad- 



Fig. 3 Intraoperative photographs demonstrating a thrombosed aneurysm (AN) in the $M_{1}$ portion of the middle cerebral artery (MCA). A: Clips were applied temporarily in the left internal carotid artery, the left $A_{1}$ portion of the anterior cerebral artery, and the $M_{2}$ portion of the MCA. B: After exposure of the AN, the anterior temporal artery (ATA), dilated $10 \mathrm{~mm}$ from the origin of $M_{1}$ and the ATA, was ligated at sites proximal and distal to the AN.

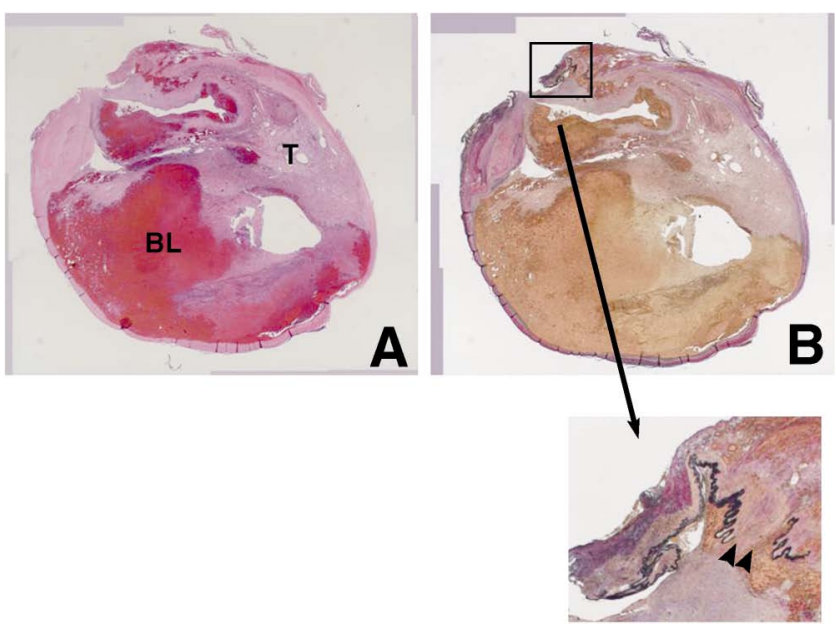

Fig. 4 Photomicrographs of the aneurysm. A: Hematoxylineosin staining showing the false lumen occluded by fresh blood $(B L)$ and thrombus $(T)$. Original magnification $\times 5 . \quad B$ : Elastica-van Gieson staining demonstrating dissection of the internal elastic lamina (arrowheads). Original magnification $\times 20$.

ual enlargement of the dissecting segment resulting in the formation of a giant or serpentine aneurysm.

Neither the indications nor the timing of surgical treatment for distal MCA dissecting aneurysms has been established due to a lack of knowledge regarding their natural history. These lesions tend to enlarge gradually and rupture eventually. ${ }^{8,14)}$ Based on a distal MCA dissecting aneurysm that resulted in intracerebral hemorrhage 4 years after its non-hemorrhagic onset, surgical treatment was strongly recommended if ectatic components are identified. ${ }^{8)}$

In our patient, preoperative angiography revealed aneurysmal dilatation and a thrombosed mass. Under a diagnosis of thrombosed aneurysm of the horizontal segment of the MCA, we performed surgery to reduce the risk of repeated infarction and hemorrhage. Intraoperatively we identified occlusive changes on the distal side of the
ATA suggestive of arterial dissection. The dissecting lesion was ligated and resected and our patient developed no new neurological deficits or additional ischemic lesions. We recommend that in such cases, acute surgery should be considered to prevent rupture and subarachnoid hemorrhage.

\section{References}

1) Abiko S, Okamura T, Kurokawa $Y$, Ikeda $N$, Ideguchi $M$, Watanabe K: [Diagnosis and treatment of nontraumatic dissecting aneurysm in the middle cerebral artery]. No Shinkei Geka 27: 743-749, 1999 (Japanese)

2) Ahn JY, Han IB, Joo JY: Aneurysm in the penetrating artery of the distal middle cerebral artery presenting as intracerebral haemorrhage. Acta Neurochir (Wien) 147: 1287-1290, 2005

3) Chokyu I, Terada T, Matsuda Y, Okumura H, Shintani A, Nakamura Y: [A case of spontaneous fusiform aneurysm in a middle cerebral artery branch which causes rapidly thrombosed formation in a short period]. No Shinkei Geka 35: 1109-1113, 2007 (Japanese)

4) Day AL, Gaposchkin CG, Yu CJ, Rivet DJ, Dacey RG Jr: Spontaneous fusiform middle cerebral artery aneurysms: Characteristics and a proposed mechanism of formation. J Neurosurg 99: 228-240, 2003

5) Hashimoto H, Iida J, Shin Y, Hironaka Y, Sakaki T: Subarachnoid hemorrhage from intracranial dissecting aneurysms of the anterior circulation. Two case reports. Neurol Med Chir (Tokyo) 39: 442-446, 1999

6) Hayashi Y, Shima H, Kinoshita M, Nakada M, Miyashita K, Hamada J: Ossified peripheral middle cerebral artery aneurysm in a 30-year-old man. J Clin Neurosci 16: 1075-1077, 2009

7) Horiuchi T, Tanaka $Y$, Takasawa H, Murata T, Yako T, Hongo K: Ruptured distal middle cerebral artery aneurysm. J Neurosurg 100: 384-388, 2004

8) Isono $M$, Abe $T$, Goda $M$, Ishii $K$, Kobayashi $H$ : Middle cerebral artery dissecting aneurysm causing intracerebral hemorrhage 4 years after the non-hemorrhagic onset: A case report. Surg Neurol 57: 346-349, 2002

9) Kawaguchi T, Kawano T, Kazekawa K, Honma T, Kaneko Y, Koizumi T, Dousaka Y: [Dissecting aneurysm of the middle cerebral artery with subarachnoid hemorrhage and brain infarction: a case report]. No Shinkei Geka 25: 1033-1037, 1997 (Japanese)

10) Lee KS, Liu SS, Spetzler RF, Rekate HL: Intracranial mycotic aneurysm in an infant: Report of a case. Neurosurgery 26: 129-133, 1990

11) Lee SH, Bang JS: Distal middle cerebral artery M4 aneurysm surgery using navigation-CT angiography. J Korean Neurosurg Soc 42: 478-480, 2007

12) Mizutani T, Miki Y, Kojima H, Suzuki H: Proposed classification of nonatherosclerotic cerebral fusiform and dissecting aneurysms. Neurosurgery 45: 253-260, 1999

13) Ohashi Y, Horikoshi T, Sugita M, Yagishita T, Nukui H: Size of cerebral aneurysms and related factors in patients with subarachnoid hemorrhage. Surg Neurol 61: 239-245, 2004

14) Piepgras DG, McGrail KM, Tazelaar HD: Intracranial dissection of the distal middle cerebral artery as an uncommon cause of distal cerebral artery aneurysm. Case report. J Neurosurg 80: 909-913, 1994

15) Rinne J, Hernesniemi J, Niskanen M, Vapalahti M: Analysis of 561 patients with 690 middle cerebral artery aneurysms: Anatomic and clinical features as correlated to management 
outcome. Neurosurgery 38: 2-11, 1996

16) Russell SM, Lin K, Hahn SA, Jafar JJ: Smaller cerebral aneurysms producing more extensive subarachnoid hemorrhage following rupture: A radiological investigation and discussion of theoretical determinants. J Neurosurg 99: 248-253, 2003

17) Saito A, Fujimura M, Inoue T, Shimizu H, Tominaga T: Lectin-like oxidized low-density lipoprotein receptor 1 and matrix metalloproteinase expression in ruptured and unruptured multiple dissections of distal middle cerebral artery: Case report. Acta Neurochir (Wien) 152: 1235-1240, 2010

18) Saito H, Ogasawara K, Kubo Y, Saso M, Otawara Y, Ogawa A: Treatment of ruptured spontaneous saccular aneurysm in the central artery of the middle cerebral artery using bypass surgery combined with trapping-case report. Neurol Med Chir (Tokyo) 47: 471-474, 2007

19) Sakamoto S, Ikawa F, Kawamoto H, Ohbayashi N, Inagawa
$\mathrm{T}$ : Acute surgery for ruptured dissecting aneurysm of the M3 portion of the middle cerebral artery. Neurol Med Chir (Tokyo) 43: 188-191, 2003

20) Takemura Y, Hirata Y, Sakata N, Nabeshima K, Takeshita $\mathrm{M}$, Inoue T: Histopathologic characteristics of a saccular aneurysm arising in the non-branching segment of the distal middle cerebral artery. Pathol Res Pract 206: 391-396, 2010

21) Yamahata $H$, Tokimura $H$, Hirabaru $M$, Hirano $H$, Arita $K$ : Aneurysm on the cortical branch ( $\mathrm{P} 4$ segment) of the posterior cerebral artery. Case report. Neurol Med Chir (Tokyo) 50: 1084-1087, 2010

Address reprint requests to: Katsuya Umeoka, MD, Department of Neurosurgery, Mitsui Memorial Hospital, 1 Kandaizumi-cho, Chiyoda-ku, Tokyo 101-8643, Japan.

e-mail: katsuya@nms.ac.jp 